\title{
How to Fly with Statistics
}

\section{WILLEM ALBERS}

To cite this article: WILLEM ALBERS (1990) How to Fly with Statistics, EUROPEAN JOURNAL OF ENGINEERING EDUCATION, 15:3, 199-204, DOI: 10.1080/03043799008939476

To link to this article: https://doi.org/10.1080/03043799008939476

$$
\text { 曲 Published online: } 15 \text { Oct } 2007 .
$$

Submit your article to this journal $\sqsubset$

\section{Џ Article views: 24}

Q View related articles $\longleftarrow$ 


\title{
How to Fly with Statistics
}

\author{
WILLEM ALBERS
}

SUMMARY A profile is sketched of engineering statistics by contrasting it with statistics courses for students of mathematics on one hand, and for social science students on the other. Aspects which play a role comprise level of abstraction, amount of technical detail and choice of subjects. A brief outline is given of feasible forms and contents of statistics courses for engineers. Special attention is devoted to the nature of exercises suitable in this context. Questions addressed concern the degree of adaptation to the student's own field of study and the (dis)advantage of embedding the pure statistical problems into a technical 'story'.

Zusammenfassung Statistik für Ingenier Studenten wird durch Vergleich von Vorlesungen ïber Statistik für Studenten der Mathematik auf der einen Seite und für Studenten der Sozialwissenschaften auf der anderen skizziert. Aspekte, welche eine Rolle spielen, umfassen das Niveau der Abstraktion, die Anzahl technischer Details und die Wahl der Themen. Eine kurze Übersicht wird gegeben über mögliche Formen und Inhalte von Vorlesungen über Statistik für Studenten der Ingenieurwissenschaften. Spezielle Beachtung wird den Übungen gewidmet Dabei wird gefragt, wie stark der Einfluss des eigenen Gebietes der Studenten auf die Art der Aufgaben sein soll, und welches die Vorund Nachteile sind, wenn die eigentliche statistische Fragestellung in eine technische 'Geschichte' eingebettet wird.

\section{Introduction}

Perhaps the best way to begin this paper is to explain its high-sounding title. One important aspect in teaching statistics is that it should never be dull. In that respect it. seems inadmissible to call a paper on statistics in engineering curricula 'Statistics in engineering curricula'. This even more so as the paper is part of a whole issue devoted to this subject. However, the reader may object, one point is that teaching should not be dull, but another criterion most certainly is that it should make sense: non-dull nonsense does not get us anywhere either.

So let me hasten to point out that the title appeals to at least one of our senses, as it echoes the well-known title 'How to lie with statistics'. This latter title clearly expresses the widespread distrust of statistics and statisticians, already present in Disraeli's days ("... lies, damned lies, statistics ...") and continuing into the present age. Originally this negative attitude solely fed on the fruits of descriptive statistics such as figures (clearly mis-)representing a data set leading to (disastrous) 'factsfrom-figures' conclusions. Later on the distrust was strengthened by the sometimes 
confusing and conflicting results of multivariate statistical approaches in problems from the social sciences.

In contradistinction, the title 'How to fly with statistics' points into the opposite direction: it is trust-inspiring (even if we allow for a considerable fear of flying). Rather than to lie with statistics, you can rely on it, which reliability comes in handy if you contemplate flying. But flying is one of the major achievements of enginering and reliability theory is an indispensable statistical tool in the design, construction and inspection of aircraft. Hence, the title chosen symbolises the role of statistics in the engineering sciences: not some dubious way of deceiving unsuspecting laymen, but instead one of the basic crafts needed to obtain constructions that are well-founded.

In Section 2 the way in which statistics is taught to enginering students is compared with the approaches used for students of mathematics on one hand and for students in the social sciences on the other. Not surprisingly, in discussing differences with mathematicians, the level of abstraction plays a key role. In comparing with the social sciences various aspects spring to mind. One issue is the amount of technical mathematical detail, another the choice of subjects after the almost obligatory first steps. Finally, the order of presentation can lead to differences.

The next section briefly discusses how much time should be, and how much in fact typically will be, allotted to statistics and moreover, which forms of teaching occur. Section 4 is devoted to a description of the subjects which almost invariably are treated in an introductory statistics course. Finally, Section 5 pays attention to the nature of statistical exercises for engineering students. For example, the question of the extent to which it makes sense to use exercises with a technical setting is addressed. This in its turn leads to the point whether it is easier to solve 'bare' mathematical problems, rather than 'real' problems which come in the form of a story.

\section{Profile of Engineering Statistics}

In this section a profile of statistics for engineering students is sketched by contrasting it to statistics courses for students of other disciplines. First consider the mathematicians. An obvious difference is that the latter group will have more courses on the subject than the technical students. However, this does not necessarily imply that they cover more ground, the point is that they are urged to dig a bit deeper, not so much to uncover more technical niceties, but rather to contemplate the underlying ideas. To illustrate this higher level of abstraction, consider a few examples.

To begin with, in the part on probability theory, the mathematical students will be exposed to the measure theoretic approach. It is hoped that they will appreciate and benefit from a unified treatment of distributions, containing the discrete and continuous ones as special cases, corresponding to counting and Lebesgue measure, respectively. For engineers, however, one is inclined to feel that the advantage of greater mathematical purity is outweighed by the simplicity of the direct approach which tackles problems one at a time: first the discrete case using point probabilities, and then the continuous case using densities. A link between the two is then made by means of a-not very satisfactory-limiting argument.

A second example is drawn from the main statistical part. For technical students, the ideas behind hypothesis testing are duly explained and it is demonstrated how a test works in general. Nevertheless, most attention is devoted to a number of important special cases, such as tests for the normal and the binomial case. For the mathematicians, the emphasis will be a bit more on the underlying principles than on the special examples. To be specific, a mathematics student will have to be able to 
derive a sensible test (e.g. likelihood ratio) for a testing problem concerning an arbitrary given family of underlying distributions. A related issue concerns confidence intervals. Engineers will often encounter the following approach: in the normal and binomial examples, start with the statistics previously used successfully in hypothesis testing. Find an interval connected with these statistics which has a sufficiently high probability and 'shake' this interval until your confidence interval comes rolling out (hopefully!). This trick-like approach is very successful in the standard examples, but is inspiring little confidence otherwise, as it does not lead to an understanding of what precisely is going on. The more illuminating, but admittedly slower, way of first demonstrating that confidence intervals can be derived from hypothesis tests (by collecting all parameter values which would not have led to rejecting the corresponding hypothesis against the appropriate alternative) is typically reserved for students of mathematics.

After the mathematicians, it is the turn of the social scientists to serve as a control group. A first distinction is the amount of technical mathematical detail. Note that this did not occur as a difference between engineers and mathematicians. In fact, if there is a difference, the winners could very well be the engineers in view of their tendency to plod on doggedly where mathematics courses might side-step to a more abstract approach avoiding technical details. Such a brave attitude towards technical obstacles is of course made possible by the fact that engineering students usually have a firm basis in calculus and matrix algebra, falling not too far short of what has been taught to their mathematical colleagues in these areas. For students in the social sciences, the situation is quite different in this respect. Usually a non-negligible percentage of these students is mathematically illiterate, in the sense that they become more or less paralysed as soon as something like an ' $e$ ' or an ' $f$ ' appears on the blackboard. (To avoid misunderstandings, there is also always a considerably large group of students which is quite talented in a mathematical respect, but the point is to determine what can be presented as an introductory course to the group as a whole). Hence a more verbal presentation is called for, highlighting the underlying ideas, suppressing the mathematical details and stressing what can, and more importantly, what cannot be expected of a certain statistical technique. The obvious, and in this situation uncanningly appropriate methaphor is that of handling a car: social scientists are instructed how to drive, technicians are also allowed to look under the hood.

In passing, note that an amusing and somewhat bewildering consequence of taking giant steps by skipping technical details is that one gets remarkably far (sometimes out on a limb): social science students within a short time use (e.g. through SPSS) rather sophisticated procedures with many even more complicated options (e.g. stepwise selection), thus generating more questions than 10 wise statisticians can answer. Another result of such a formidable speed is that sometimes remedial teaching is called for: a real-life example is $\mathrm{Ph}$.D. students having to take an elementary course in matrix algebra, as it becomes progressively embarrassing to use criteria for choosing between, for example, regressors based on 'things' like eigenvalues, traces and determinants, when one has no idea what a matrix is!

A second distinction between courses for social science students and engineering students is also rather obvious: the choice of subjects treated differs. The introductory steps agree, since both groups will need to know the basic ideas behind point and interval estimation and testing as applied to one- and two-sample normal and binomial distributions. But after this obligatory part, divergence will occur. For technical students, methods are relevant which are designed for the analysis of research in relatively well-controlled circumstances such as laboratory experiments. Hence regres- 
sion, and in particular ANOVA, will be of interest. In social science on the other hand, a lot of research is necessarily observational rather than experimental. This leads among others to 'fishing-net' methods such as cluster and factor analysis where one tries to discover structure in large data sets.

A final difference to be mentioned here is the order of presentation. For engineers, the same approach is usually chosen as for mathematicians in this respect. First a basis in calculus and matrix algebra is administered. On this, an introduction to probability can be built, after which the final step of a statistics course can follow. For social science students, a different approach is often preferred. For them, an order dictated by mathematical convenience typically is not very inspiring. Their interest in statistics solely stems from the desire to analyse data and hence it may be preferable to take this desire as a starting point. Hence some descriptive statistics is first presented, such as graphical techniques and descriptive measures. Then students can start analysing data right away, and immediately obtain some results from statistics. Since the descriptive techniques are merely exploratory, the students are automatically lured further into statistics, as the desire to draw conclusions from the pictures and/or measures obtained remains to be fulfilled. Hence at this point some probability theory is called for, after which results on inferential statistics can be presented.

\section{Size and Form of Programme}

In this section some brief remarks are devoted to external factors such as size and form of the programmes before moving to the actual contents of statistics courses for engineers. Invariably it will be felt that too little time is available for statistics and that many interesting subjects cannot be covered due to this lack of time. But similar feelings are uniformly present in all departments, especially in The Netherlands since the curriculum was shortened to four years. Hence it does not help much to complain about this state of affairs, so let us just concentrate on what is possible. Usually, about two quarters of a year are available, in which about four hours a week are devoted to teaching.

Regarding the form, the basic variant consists of a plenary two-hour course in which the theory is explained together with a two-hour exercises session in smaller groups of about 25 students. An alternative is to work with such small groups throughout and to have integrated sessions in which attention for theory and exercises alternates more frequently. Obviously, this alternative is more expensive, but in some situations it can be a valuable investment. Statistics quite often is one of the more difficult subjects in the curriculum and delays for considerable amounts of students can possibly be avoided by offering a more than average intensity of instruction.

\section{Topics to be Treated}

In sketching the profile of engineering statistics in Section 2, already quite a lot has been said about the contents of the programme. Here a more systematic account will be given. In this way it is hoped to avoid the presentation of a variety of comments about engineering statistics courses without being at all clear about what the actual content of such a programme is supposed to be. On the other hand, presentation of a dull listing of key words should be avoided, and hence the survey will be kept rather global.

First consider the part on probability theory. The notion of probability is introduced, using subjective probabilities, Laplace's definition and the frequency interpreta- 
tion as stepping stones towards the axioms of Kolmogorov. The basic rules for calculation of probabilities are presented. Combinatorics comes next, but should be kept relatively simple. Considerable attention is required for conditional probabilities, Bayes' rule and independence. The next step leads to random variables and distributions. First discrete distributions such as the binomial, Poisson and geometric are introduced. Then continuous distributions and densities follow. For engineering students it is necessary to treat not only the normal case, but certainly also the exponential. After the distributions of one variable the simultaneous case is treated, followed by moments and correlation. If time allows, a short treatment of characteristic functions might be advisable. Some attention should be devoted to the central limit theorem, but this should be better applied, concentrating, for example, on the normal approximation to the binomial. Discussion of convergence in a more general context does not seem too rewarding. Some students only remember that there seems to be a 'large law of weak numbers'!

Next the main course, statistics. First there is a kind of border area between probability and statistics, in which distributions like $\chi^{2}, t$ and $F$ are introduced. Then the notions of a random sample and of a statistic follow, with some examples of the latter, such as sample mean and variance. As a first statistical technique, point estimation is next. The concepts of unbiasedness and consistency should be covered, as well as the method of maximum likelihood. Applications will involve typically the normal and binomial cases, with the exponential as a suitable third candidate. Then it is time to explain the principles of hypothesis testing and to apply these to one- and two-sample normal and binomial situations, usually followed by a derivation of the corresponding confidence intervals. This more or less concludes the absolutely required minimum. Almost standard by now will also be some attention to nonparametric methods such as the sign test and Wilcoxon's test. Another likely topic is that of the $\chi^{2}$-test for goodness of fit. As explained in Section 2, the remaining time should be devoted to regression and analysis of variance (ANOVA).

\section{Nature of Exercises}

In this final section some remarks will be made on exercises. After the enumeration of subjects to be treated in the courses on statistical theory, the reader probably has little appetite left for another such account on exercises. This provides the liberty to resort to just pointing out a few special aspects of exercises in an engineering context.

To begin with, there is the question of what extent exercises should be adapted to the particular type of student for which they are destined. The receiving departments tend to go quite far in this respect. In working with sampling with or without replacement, the electrical engineers would like the statisticians to come up with boxes filled with transistors whereas the mechanical engineers feel that motivation is improved by boxes containing bolts, etc. However, it seems quite clear that such a literal interpretation of adaptation to special interests does not really help the understanding, and is mainly of a cosmetic nature: it 'looks' applied. One could even argue that it is dangerous to require too specific a coating, as it prevents the student from gaining even the slightest feeling for abstraction. The familiar coat then becomes a strait-jacket.

To elaborate a little more on this point, an analogous controversy arises concerning notational problems. Of course, notation should be well-chosen and it may well serve to help remember a bit of theory, just as rhyme can help to remember a poem. But students should not become dependent on it. Hence, as an example consider the 


\section{W. Albers}

binomial distribution with parameters $n$ and $p$, where $n$ stands for the number of trials and $p$ for the probability of success in a single trial. Nobody forbids to consider an application where the natural number $p$ stands for the number of trials and the real number $n(0 \leq n \leq 1)$ represents the probability of success. But it will definitely be considered bad practice to do so! However, now suppose that in a certain exercise one winds up with $K$ independent trials, each of which has probability of success say $\Phi(\theta)$, where $\Phi$ is the standard normal distribution function and $\theta$ some arbitrary parameter. Then the instructor will feel some frustration if the student only recognizes the binomial distribution after being told to call $K=n$ and $\Phi(\theta)=p$.

In the above, a form of adaptation of exercises was discussed which is believed to be of little help (or worse). Obviously a better way of adapting is to let the exercises be inspired by the types of statistial problems the technical student will encounter when working in his or her field. Simple and standard examples can be found in quality control. Consider for example a Bayes' rule exercise based on a few factories producing say radios, with a varying percentage of defectives per factory. Given a defective product, the question then is with what probability did it come from a given factory? This type of exercise has been around almost as long as Bayes' rule itself (or so it seems). It only needs to be updated from time to time, 'radios' become '(colour) TVs', which in their turn become 'videos', etc.

A related question of some relevance is how much wording is helpful or useful. Hence the issue now is not whether the 'story' of the exercise should be oriented towards the receiving department, or that any story involving dice, decks of cards, birthdays, etc. will do just as well. No, the point is whether a story should be used at all. The receiving departments typically tend to feel that this definitely is the case. A bare mathematical exercise, stripped of all colouring, running like: 'Let $X$ and $Y$ be random variables from a ..., etc.' does not stir the imagination of the student. It is dull and lifeless to the non-mathematician and, they feel, not motivating. There is undoubtedly much truth in this observation. Unfortunately, by adding a story one may get from the frying-pan into the fire. The exercise becomes lively, motivating, but also a lot harder to solve. The original mathematical problem is still sitting there, waiting to be solved, but first it has to be unravelled from the story in which it is now enclosed. It is precisely this combination of mathematical and real-life problems which makes statistics one of the more difficult subjects for many students.

The above is not meant to suggest that one should stick to purely mathematical exercises. Probably the best thing to do is to strive for a careful balance: first give a few bare exercises to get the knack of the mathematics involved. Then insert some colourful exercises to keep the motivation up by showing where it is all leading to. 